\title{
Menopausal symptoms assessment among middle age women in Kushtia, Bangladesh
}

Shahedur Rahman ${ }^{1 *}$, Faizus Salehin ${ }^{2}$ and Asif $|q b a|^{1}$

\begin{abstract}
Background: There are few menopausal study data available in South East Asia especially in Bangladesh. This study was conducted in a well populated town named Kushtia, which is located in the western part of Bangladesh.

Objectives: This study was aimed to document the menopausal-related symptoms among middle age women of Kushtia region of Bangladesh.

Methods: By using modified MRS (Menopause Rating Scale) questionnaire, 509 women aged 40-70 years were interviewed to document symptoms commonly associated with menopause.

Findings: The mean age of menopause was 51.14 years. The most prevalent symptoms reported include, feeling tired (92.90\%); headache (88.80\%); joint and muscular discomfort (76.20\%); physical and mental exhaustion (60.90\%) and sleeplessness (54.40\%) which are followed by depressive mood (37.30\%); irritability (36\%); dryness of vagina (36\%); hot flushes and sweating (35.80\%); anxiety (34.20\%). However, noted less frequent symptoms were sexual problem (31.20\%); cardiac discomfort (19.10\%) and bladder problem (12.80\%).

Conclusions: The prevalence of menopausal symptoms found in this study correspond to other studies on Asian women however the prevalence of classical menopausal symptoms of hot flushes and sweating were lower compared to studies on Caucasian women.
\end{abstract}

\section{Background}

Modern medicine has significantly prolonged human lifespan [1]. All women who live long enough will make transition to menopause [2]. Menopause which is defined as complete cessation of menstruation for twelve months or more is a normal physiological change experienced by middle age women. Some of menopausal symptoms experienced by these women can be severe enough to affect their normal lifestyle. Unfortunately majority of these women are not aware of the changes brought about by menopause [3-5]. The common climacteric symptoms experienced by them can be group into: vasomotor, physical, psychological or sexual complaints. It was also noted in some postmenopausal women with long term estrogen deficiency, changes to the cardiovascular or bone which leads to osteoporosis. It is well documented that menopausal symptoms

\footnotetext{
* Correspondence: shahed.rajib@gmail.com

'Department of Biotechnology and Genetic Engineering, Islamic University, Kushtia-7003, Bangladesh

Full list of author information is available at the end of the article
}

experienced by women affect their quality of life [6]. In Western countries, menopausal symptoms such as hot flushes, sweating and vaginal dryness are considered as the main climacteric complaints. In other cultures, these symptoms dramatically vary from those observed in Western women [7], while North American and European samples were reported higher rates of symptoms than that of Asian women [8,9]. It has been suggested that Asian women suffer more from the atypical symptoms and fewer, and with lesser severity, the typical psychological and vasomotor symptoms in comparison to those reported in Caucasian women in the west $[6,7]$. These symptoms are directly resulted from depletion of estrogen level as women approaches menopausal stage and some of these women begin to experiences these menopausal symptoms early in the perimenopausal phase [3].

Studies show that perimenopausal and postmenopausal women have more menopausal complaints than that of premenopausal women. They were noted to complain 
significantly more of vasomotor, sexual and psychological symptoms compared to premenopausal women [10,11].

Although menopause related symptoms have been extensively studied in the western countries, few data are available in Asia especially in South East Asia [3] including Bangladesh. This study was conducted in Kushtia, which is located in the western part of Bangladesh. This study aimed to document the menopausalrelated symptoms among middle age women of Kushtia region of Bangladesh.

\section{Methods}

\section{Participants and Setting}

This is a cross-sectional study. Conducted from the month of April 2010 to August 2010. This study was approved by University of Development Alternative Research and Ethics Committee. The questionnaire based interview was carried out among women ages of 40 to 70 years who visited the government and private healthcare centers in Kushtia, Bangladesh. Five hundred and sixty participants were approached among them five hundred and nine participants take part in the study. The inclusion criteria consisted of women between the ages of 40 to 70 years who gave written consent to participate in this study. Pregnant and breast feeding women, women with uncontrolled medical conditions such as hypertension, diabetes mellitus or heart disease, who were undergoing treatment for serious diseases like cancer, women who were in remission, who had history of drug or alcohol abuse and who were on hormone replacement therapy were excluded from the study.

\section{Study Questionnaire}

The questionnaire used in this survey was based on the Menopause Rating Scale (MRS) [3,9]. Questionnaire was modified to suit the Bangladeshi culture and norms. These changes allowed the researchers to cover all the profession groups in the female population living in the city, such as housewives, workers, teachers, engineers, adjudicators etc. Reliability analysis was performed on the modified Menopause Rating Scale questionnaires with Cronbach's alpha of somatic subscale 0.712, psychological subscale 0.743 and urogenital subscale 0.821 . Therefore, this study determined the prevalence of menopausal symptoms and not the severity of the symptoms.

Questionnaires were divided into three sections:

(1) Socio-demographic data of the participants, which included: age, race, religion, marital status, educational level, occupation and average household income.

(2) Menopausal status of the participants: The menopausal status was classified according to STRAW (Stages of Reproductive Aging Workshop) classification which divided menopause staging into: Postmenopausal; no menstrual bleeding in the previous/last 12 months. Late perimenopause; had menstruation in the previous/last 212 months but not in the previous/last 2 months. Early perimenopause; had increasing irregularity of menses without skipping periods (7 days difference from the beginning of a given cycle to the next, experienced after the previously regular cycle) and Premenopause; minor changes in cycle length particularly decreasing length of the cycle. To aid statistical analysis, the early and late perimenopausal transition stages were grouped together as perimenopausal stage.

(3) Menopause Rating Scale (MRS) questionnaire were used as a basis for assessing menopausal symptoms in this study, this is a self-administered instrument which has been widely used and validated and have been used in many clinical and epidemiological studies, and in research on the etiology of menopausal symptoms to assess the severity of menopausal symptoms [12].

The questionnaire was first translated into Bengali language by a group of experienced healthcare workers and language experts, and then translated back to English to validate that the original meaning of the questionnaire was maintained in the translation. Moreover, a pilot study was done on 30 women to validate the translated questionnaires. The women were asked whether or not they had experienced the menopausal symptoms shown in the questioner in the previous one month (30 days). However, it was noted from the pilot study, these women had difficulties in rating the scale themselves, in order to minimize these difficulties, a face-to-face interviewed was done rather than using self-administered respond.

All women were interviewed in Bengali language. Face to face interviews were done on all the women by trained healthcare personnel who had undergone training, as this was important to make sure right answer were given and explanations can be given if the women were in doubt or unclear about the questions asked. At the reception desk during registration, women who fulfill the criteria were invited to participate in this study. Explanations were given and written informed consent was taken from them.

\section{Statistical Analysis}

The Statistical Package for the Social Sciences software Version 14.0 (SPSS, Chicago, IL) was used for univariate analyses. The $X^{2}$ test was applied to compare the frequencies of the symptoms among the different menopausal status. The level $\mathrm{P}<0.05$ was considered as the cut-off value for significance.

\section{Results}

Five hundred and nine women took part in the study. The mean age of respondents in this study was $54.50 \pm 5.70$ 
(SD) years. And the mean age at menopause was 51.14 \pm 2.11 (SD) years. Among these women, 23.96\% were premenopausal, $42.43 \%$ perimenopausal and $33.59 \%$ postmenopausal. Most of the women studied were Muslim (90.56\%), some were Hindu (8.64\%) and very few were from other Religions $(0.78 \%)$. On the contrary, the percentage of widowed-divorced was much lower $(21.41 \%)$ than that of married women $(77.40 \%)$, but very few of them were unmarried $(1.17 \%)$. In addition, the proportion of women who received nonacademic education was the highest (48.33\%), whereas the lowest proportion of women was highly educated (5.5\%). However, $85.06 \%$ of the women were housewives and $14.93 \%$ of women were involved with paid work. The highest percentage of women were from families with average income, whereas lowest were from families with high income and figures were $66.29 \%$ and $14.14 \%$ respectively. Moreover, the proportion of women who do regular physical exercise were much lower $(21.02 \%)$ than that of who do not do any kind of exercise (44.40\%). The lion share of the studied women claimed their health as average $(50.09 \%)$ and a much lower percentage of women rated their health poor or very poor (11.98\%).

Table 1 shows the detail frequency of menopausal symptoms. The five most common menopausal symptoms for all women $(\mathrm{n}=509)$ were: Feeling tired $(92.90 \%)$, headache $(88.80 \%)$, joint and muscular discomfort (76.20\%), physical and mental exhaustion (60.90\%) and sleeplessness (54.40\%). Table 2 shows the percentage of menopausal symptoms on individual menopausal status.

\section{Discussion and Conclusion}

The age of natural menopause has been examined in several North American studies and compared to that

Table 1 Frequency of menopausal symptoms among Bangladeshi women aged 40-70 years in Kushtia

\begin{tabular}{ccc}
\hline Symptoms & $\mathbf{n = 5 0 9}$ & $\mathbf{1 0 0 \%}$ \\
\hline 1. Dryness of vagina & 183 & 36 \\
2. Joint and muscular discomfort & 388 & 76.2 \\
3. Physical and mental exhaustion & 310 & 60.9 \\
4. Sleeping problems & 277 & 54.4 \\
5. Hot flushes, sweating & 182 & 35.8 \\
6. Irritability & 183 & 36 \\
7. Anxiety & 174 & 34.2 \\
8. Depressive mood & 190 & 37.3 \\
9. Sexual problems & 159 & 31.2 \\
10. Heart discomfort & 97 & 19.1 \\
11. Bladder problems & 65 & 12.8 \\
12. Headache & 452 & 88.8 \\
13. Feeling tired & 473 & 92.9 \\
\hline
\end{tabular}

reported from other regions of the world [13]. The mean age at menopause in this study was $51.14 \pm 2.11$ years. This is slightly higher than studies done in Singapore (49.1 years), and Thailand (48.7 years) $[14,15]$. However, comparing with other research our findings still falls between the normal ranges of menopausal age $[6,13,16,17]$.

In our study, the classical presentation of menopausal symptoms; feeling tired (92.9\%) and headache $(88.8 \%)$ which is quite similar when compare to other study [9]. The other classical presentation of menopausal symptoms; hot flushes, sweating and night sweats were noted to be lower $(35.8 \%)$ in comparison to findings from studies done on western women which were reported to be from $45 \%$ to $75 \%$. However, our findings of low menopausal classical symptoms were shared by studies done in other Asian countries $[6,17]$.

From our study, joints and muscular discomfort; physical and mental exhaustions and sleeping problems (Table 2) were experienced most by perimenopausal followed by postmenopausal women and these were also statistical significant differences in comparison to premenopausal women. These findings were also noted to be corresponding to studies conducted among Asian and Caucasian women [3,18-21]. Frequency of sexual problems, bladder problems and vaginal dryness were experienced mainly by perimenopausal and postmenopausal group of women and it was also significant statistically in comparison to other menopausal status and similar finding were documented from other studies $[14,6,18,21]$.

There are some limitations of this study. As this was a cross sectional study, it does not exclude other confounding effects of the natural aging process that may influence experience of symptoms. This study used modified MRS questionnaire, translated to Bengali, so there is a question of accuracy in translation, though this was done by a group of healthcare-workers and language experts. Although MRS is a self reporting questionnaire and substantial number of women studied does not have formal education, thus in orders to include these illiterate women, interviews were used instead. Moreover, some subjects could have been misclassified into the incorrect menopause status group.

\section{Consent}

The study was performed with informed consent and following all the guidelines for experimental investigations required by the Institutional Research and Ethics Committee. 
Table 2 Percentage of menopausal symptoms in the participants according to menopausal status

\begin{tabular}{lllll}
\hline Menopausal symptoms & All & Premenopausal & Perimenopausal & Postmenopausal \\
\hline Dryness of vagina & 36 & 11.4 & $43.9^{*}$ & $43.2^{* * \neq}$ \\
Joint and muscular discomfort & 76.2 & 35.2 & $88.88^{*}$ & $86.4 \neq$ \\
Physical and mental exhaustion & 60.9 & 40.1 & 69.44 & $64.9 \neq$ \\
Sleeping problems & 54.4 & 29.5 & $58.7^{*}+$ & 52.6 \\
Hot flushes, sweating & $35.8^{*}$ & 27.8 & 37.5 & 39.1 \\
Irritability & 36 & 29.5 & $38.8^{*}+$ & $36.8 \neq \dagger$ \\
Anxiety & 34.2 & 25.4 & $35.6+$ & 38.59 \\
Depressive mood & 37.3 & 30.3 & 35.6 & $44.4 \neq$ \\
Sexual problems & 31.2 & 18.8 & $35.1+$ & $35 \neq$ \\
Heart discomfort & 19.1 & 11.4 & $25.9^{*} \dagger$ & 15.7 \\
Bladder problems & 12.8 & 5.7 & 14.8 & $15.2^{* * \neq}$ \\
Headache & 88.8 & 77.86 & $91.2 \dagger$ & 93.5 \\
Feeling tired & 92.9 & 88.5 & 94.4 & 94.1 \\
\hline
\end{tabular}

* Significant difference $p<0.05$ compared to premenopausal.

† Significant difference $\mathrm{p}<0.05$ compared to postmenopausal.

₹ Significant difference $\mathrm{p}<0.05$ compared to premenopausal.

** Significant difference $p<0.05$ compared to perimenopausal.

\section{Acknowledgements}

We thank all the participants who take part in this study.

\section{Author details}

${ }^{1}$ Department of Biotechnology and Genetic Engineering, Islamic University, Kushtia-7003, Bangladesh. ²Department of Biotechnology and Genetic Engineering, University of Development Alternative, Dhaka, Bangladesh.

\section{Authors' contributions}

SR participated in the design, coordinating and carried out the study and drafted the manuscript. FS and Al performed the statistical analysis and drafted the manuscript. All authors read and approved the final manuscript.

\section{Competing interests}

The authors declare that they have no competing interests.

Received: 14 December 2010 Accepted: 15 June 2011

Published: 15 June 2011

\section{References}

1. Changa MH, Wangb SJ, Wanga PH, Fuhd JL: Attitudes towards Menopause among Middle-Aged Women: A Community Survey In an Island Of Taiwan. Maturitas 2005, 52:348-355

2. Wang SJ, Lue SR, Juang KD, Chiu LM: The Kinmen women-health investigation (kiwi): a menopausal study of \& population aged 40-54 Maturitas 2001, 39:117-120.

3. Rahman SASA, Zainudin SR, Kar Mun VL: Assessment of menopausal symptoms using modified Menopause Rating Scale (MRS) among middle age women in Kuching, Sarawak, Malaysia. Asia Pacific Family Medicine 2010, 9:5.

4. Williams RE, Levine KB, Kalilani L, Lewis J, Clark RV: Menopause-specific questionnaire assessment in US population-based study shows negative impact on health-related quality of life. Maturitas 2009, 62:153-159.

5. Lu J, Liu J, Eden J: The experience of menopausal symptoms by Arabic women in Sydney. Climacteric 2007, 10:72-77.

6. Dhillon HK, Singh HJ, Rashidah S, Abdul Manaf H, Nik Mohd Zaki NM: Prevalence of menopausal symptoms in women in Kelantan, Malaysia. Maturitas 2006, 54:213-221.

7. Fuh Jl, Wang SJ, Lu SR, Juang KD, Chiu LM: The Kinmen women-health investigation (KIWI): a menopausal study of a population aged 40-54. Maturitas 2001, 39:117-24.

8. Dennerstein L: Well-being, symptoms and the menopausal transition. Maturitas 1996, 23:147-57.
9. Ayranci U, Orsal O, Orsal O, Arslan G, Emeksiz DF: Menopause status and attitudes in a Turkish midlife female population: an epidemiological study. BMC Women's Health 2010, 10:1.

10. Harvey C, Bee HT, Chia CA, Ee MC, Yap SC, Seang MS: The prevalence of menopausal symptoms in a community in Singapore. Maturitas 2002, 41:275-282.

11. Lori AB, Crystal MS, Kavita N: Is This Women Perimenopausal. JAMA 2003, 289(7).

12. Heinemann LAJ, Potthoff $P$, Schneider HP: International version of the menopause rating scale (MRS). Health Qual Life Outcomes 2003, 1:28.

13. Palacios S, Henderson WW, Siseles N, Tan D, Villaseca P: Age of menopause and impact of climacteric symptoms by geographical region. Climacteric 2010, 13:419-428.

14. Peeyananjarassri K, Cheewadhanaraks S, Hubbard M, Zoa Manga R, Manocha R, Eden J: Menopausal Symptoms in a hospital-based sample of women in southern Thailand. Climacteric 2006, 9:23-29.

15. Boulet MJ, Oddens BJ, Lehert P, Verme HM, Visser AP: Climacteric and menopause in seven South-east Asian countries. Maturitas 1994, 19:157-76.

16. Gold EB, Bromberger J, Crawford S, et al: Factors associated with age at natural menopause in a multiethnic sample of midlife women. Am J Epidemiol 2001, 153:865-74.

17. Ismail NN: A study on menopause in Malaysia. Maturitas 1994, 19:205-209.

18. Loh FH, Khin LW, Saw SM, Jeannette JM, Ken Gu: The age of menopause and the menopause transition in a multiracial population: a nation-wide Singapore study. Maturitas 2005, 52:169-180.

19. Lam PM, Leung TN, Haines C, Chung TK: Climacteric symptoms and knowledge about hormone replacement therapy among Hong Kong Chinese women aged 40-60 years. Maturitas 2003, 45:99-107.

20. Gold EB, Block G, Crawford S: Lifestyle and demographic factors in relation to vasomotor symptoms: baseline results from the Study of Women's Health Across the Nation. Am J Epidemiol 2004, 159:1189-1199.

21. Park YJ, Kim HS, Chang SO, Kang HC, Chun SH: Sexuality and related factors of postmenopausal Korean women. Taehan Kanko Hakhoe Chi 2003, 33:457-63.

doi:10.1186/1756-0500-4-188

Cite this article as: Rahman et al:: Menopausal symptoms assessment among middle age women in Kushtia, Bangladesh. BMC Research Notes 2011 4:188. 